RICHARD DEDEKIND

Uber die Theorie der

ganzen algebraischen Zahlen 


\section{RICHARD DEDEKIND}

\section{Uber die Theorie der ganzen algebraischen Zahlen}

Mit einem Geleitwort von

B. VAN DER WAERDEN

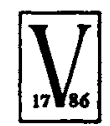

FRIEDR. VIEWEG \& SOHN - BRAUNSCHWEIG 1964 
Die vorliegende Ausgabe ist ein Nachdruck des Flften Supplements von DIRICHLETs Vorlesungen über Zahlentheorie, 4. Auflage, in den Fassungen XLVI bis XLIX nebst den Erlănterungen von $\mathrm{E}$. Noether, entnommen aus Richard Dedekind, Gesammelte mathematische Werke, Dritter Band, Braunschweig 1932 


\section{Geleitwort}

Zur Rechtfertigung dieser Edition von Dedekinds berühmtem elften Supplement zu Dirichlets „Vorlesungen über Zahlentheorie" kann ich keine besseren Worte finden als die von Dedekind selbst am Schluß seines Vorworts zur zweiten Auflage dieser „Vorlesungen“"(1871):

„Endlich habe ich mich bemüht, überall, wo es mir möglich war, auf die Quellen zu verweisen, um den Leser zum Studium der Originalwerke zu veranlassen und in ihm ein Bild von den Fortschritten der Wissenschaft zu erwecken, deren ebenso tiefe wie erhabene Wahrheiten einen Schatz bilden, welcher die unvergängliche Frucht eines wahrhaft edelen Wettkampfes der europäischen Völker ist."

Das elfte Supplement, das zuerst in der dritten Auflage erschien, war eine Neufassung eines bedeutenden Abschnittes (\$§ 159-170) des zehnten Supplementes der zweiten Auflage. Über diesen Abschnitt schreibt Dedekind im Vorwort zur zweiten Auflage:

,Endlich habe ich in dieses Supplement eine allgemeine Theorie der Ideale aufgenommen, um auf den Hauptgegenstand des ganzen Buches von einem höheren Standpunkte aus ein neues Licht zu werfen; hierbei habe ich mich freilich auf die Darstellung der Grundlagen beschränken müssen, doch hoffe ich, daß das Streben nach charakteristischen Grundbegriffen, welches in anderen Teilen der Mathematik mit so schönen Erfolgen gekrönt ist, mir nicht ganz mißglückt sein möge."

Schon vor Dedekind hatte Kronecker eine Idealtheorie der algebraischen Zahlkörper entwickelt, aber die Dedekindsche Theorie ist unabhängig von der Kroneckerschen entstanden. Dedekind fährt nämlich fort:

„Die Untersuchungen in diesem von Kummer geschaffenen Gebiete, welche Kronecker vor vierzehn Jahren angestellt hat, sind bis jetzt nicht veröffentlicht, und ich vermag nach den damaligen brieflichen Mitteilungen dieses ausgezeichneten Mathematikers nicht zu beurteilen, in welchen Beziehungen seine Prinzipien zu den meinigen stehen."

Die Dedekindsche Idealtheorie in ihrer ursprünglichen Form ist in $\$ \$ 159-$ 163 des zehnten Supplementes der zweiten Auflage von Dirichlets Zahlentheorie zum ersten Male dargestellt. Dieser Abschnitt ist als Abhandlung XLVII in die vorliegende Publikation aufgenommen.

In der französisch geschriebenen Abhandlung XLVIII vom Jahre 1877 hat Dedekind die Theorie nach seinen eigenen Worten ,,ausführlicher und in etwas veränderter Form dargestellt". Die französische Abhandlung enthält viele Beispiele und hat dadurch mehr den Charakter einer 
elementaren Einführung. Der Aufbau des elften Supplementes der dritten Auflage ist aus der französischen Abhandlung übernommen. Ferner enthält die dritte Auflage ein Stück allgemeine Idealtheorie, nämlich die eindeutige Zerlegung der Ideale einer Ordnung in ,einartige Ideale". Dieses Stück ist als Abhandlung XLIX in die vorliegende Publikation aufgenommen. Ein Beweis, den Dedekind für die dritte Auflage kassiert hatte, wurde von Emmy Noether im Nachlaß gefunden und an der betreffenden Stelle wieder eingefügt.

Im elften Supplement der vierten Auflage (1894) hat Dedekind die Theorie ganz neu aufgebaut. Bei der Edition des dritten Bandes der gesammelten mathematischen Werke von Dedekind hat Emmy Noether die Fassung der vierten Auflage vollständig aufgenommen, während von den früheren Fassungen nur jeweils das dort nicht übernommene gebracht wurde. Diese vorzügliche Anordnung wurde in dieser Publikation beibehalten. Die Erläuterungen von Emmy Noether findet man am Schluß der Abhandlung XLIX.

Über die Entstehungsgeschichte des Supplementes zur 2. Auflage ist mir nicht mehr bekannt als das wenige, was Dedekind in seiner Abhandlung XV „Über den Zusammenhang zwischen der Theorie der Ideale und der Theorie der höheren Kongruenzen" (Abh. Ges. Wiss. Göttingen 23, 1878) mitteilt, nämlich :

„,Die neuen Prinzipien, durch welche ich zu einer ausnahmelosen und strengen Theorie der Ideale gelangt bin, habe ich zuerst vor sieben Jahren in der zweiten Auflage der Vorlesungen über Zahlentheorie von Dirichlet $(\S \S 159-170)$ entwickelt und neuerdings in dem Bulletin des sciences mathématiques et astronomiques (t. XI, p. 278; t. I ( $2^{\mathrm{e}}$ série), p. 17, $69,144,207)$ ausführlicher und in etwas veränderter Form dargestellt. Mit demselben Gegenstand hatte ich mich schon vorher, durch die große Entdeckung Kummers angeregt, eine lange Reihe von Jahren hindurch beschäftigt, wobei ich von einer ganz anderen Grundlage, nämlich von der Theorie der höheren Kongruenzen ausging: allein obgleich diese Untersuchungen mich dem erstrebten Ziele sehr nahe brachten, so konnte ich mich zu ihrer Veröffentlichung doch nicht entschließen, weil die so entstandene Theorie hauptsächlich an zwei Unvollkommenheiten leidet. Die eine besteht darin, daß die Untersuchung eines Gebietes von ganzen algebraischen Zahlen sich zunächst auf die Betrachtung einer bestimmten Zahl und der ihr entsprechenden Gleichung gründet, welche als Kongruenz aufgefaßt wird, und daß die so erhaltenen Definitionen der idealen Zahlen (oder vielmehr der Teilbarkeit durch die idealen Zahlen) zufolge dieser bestimmt gewählten Darstellungsform nicht von vornherein den Charakter der Invarianz erkennen lassen, welcher in Wahrheit diesen Begriff zukommt; die zweite Unvollkommenheit dieser Begründungsart besteht darin, daß bisweilen eigentümliche Ausnahmefälle auftreten, welche eine besondere Behandlung verlangen. Meine neuere Theorie dagegen gründet sich ausschließlich auf solche Begriffe, 
wie die des Körpers, der ganzen Zahl, des Ideals, zu deren Definition es gar keiner bestimmten Darstellungsform der Zahlen bedarf, und wie hierdurch der erstgenannte Mangel von selbst wegfällt, so bewährt sich die Kraft dieser äußerst einfachen Begriffe auch darin, daß bei dem Beweise der allgemeinen Gesetze der Teilbarkeit eine Unterscheidung mehrerer Fälle gar niemals mehr auftritt".

Über die Entstehung der definitiven Fassung des elften Supplementes in der vierten Auflage von 1894 und über die Beziehung der Dedekindschen Begründungen zur Kroneckerschen. die im Frühjahr 1888 endlich publiziert wurde, weiß man viel mehr. Dedekind selbst hat nämlich in einer Abhandlung ,Ủber die Begründung der Idealtheorie" (Nachr. Ges. Wiss. Göttingen 1895, S. 106-113 =- Werke II. S. 50-58) zu diesen Fragen Stellung genommen.

Das elfte Supplement von Dedekind hat in seinen drei Fassungen eine nachhaltige Wirkung ausgeübt. Es markiert einen Wendepunkt in cler Geschichte der Zahlentheorie und der Algebra. Der 1897 in Bd. 4 des Jahresberichtes der D.M.V. erschienene ,Zahlbericht" von Hilbert zeigt, wie sich die Theorie der algebraischen Zahlkörper auf dem von Dedekind geschaffenen Fundament in kurzer Zeit zu einer erstaunlichen Höhe entwickelt hat. Für Emmy Noether war das elfte Supplement eine unerschöpfliche Quelle von Anregungen und Methoden. Bei jeder Gelegenheit pflegte sie zu sagen ,Es steht schon bei Dedekind".

Evariste Galois und Richard Dedekind sind es, die der modernen Algebra ihre Struktur gegeben haben. Das tragende Skelett dieser Struktur stammt von ihnen.

Zürich. 1. November 1963

B. L. van der Waerden 


\section{Inhaltsverzeichnis}

XLVI. Uber die Theorie der ganzen algebraischen Zahlen

(Supplement XI von Dirichlets Vorlesungen äbcr Zahlentheorie, 4. Aufl., S.434-657 (1894).)

\$159. Theorie der komplexen ganzen Zahlen von Gauß ........... 2

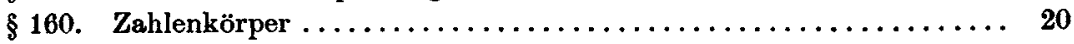

$\$ 161$. Permutation eines Körpers $\ldots \ldots \ldots \ldots \ldots \ldots \ldots \ldots \ldots \ldots \ldots \ldots .24$

162. Resultanten von Permutationen $\ldots \ldots \ldots \ldots \ldots \ldots \ldots \ldots \ldots \ldots .29$

$\S$ 163. Multipla und Divisoren von Permutationen $\ldots \ldots \ldots \ldots \ldots \ldots \ldots, 30$

$\$ 164$. Irreduzible Systeme. Endliche Körper $\ldots \ldots \ldots \ldots \ldots \ldots \ldots \ldots \ldots .33$

$\$ 165$. Permutationen endlicher Körper .................... 41

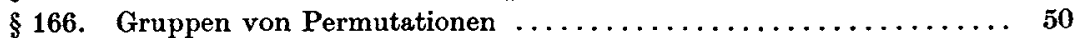

$\S 167$. Spuren, Normen, Diskriminanten $\ldots \ldots \ldots \ldots \ldots \ldots \ldots \ldots \ldots \ldots \ldots$

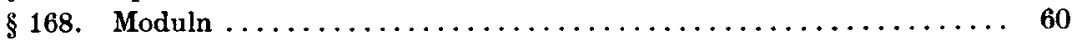

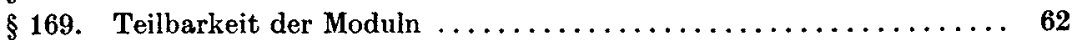

$\$ 170$. Produkte und Quotienten von Moduln. Ordnungen .......... 67

$\S 171$. Kongruenzen und Zahlklassen $\ldots \ldots \ldots \ldots \ldots \ldots \ldots \ldots \ldots \ldots \ldots \ldots$

$\$$ 172. Endliche Moduln $\ldots \ldots \ldots \ldots \ldots \ldots \ldots \ldots \ldots \ldots \ldots \ldots \ldots \ldots, 80$

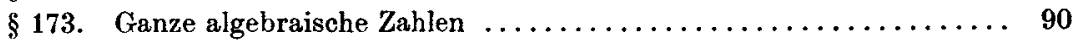

\$174. Teilbarkeit der ganzen Zahlen $\ldots \ldots \ldots \ldots \ldots \ldots \ldots \ldots \ldots \ldots \ldots .98$

$\$$ 175. System der ganzen Zahlen eines endlichen Körpers ........... 101

\$176. Zerlegung in unzerlegbare Faktoren. Ideale Zahlen . . . . . . . 107

$\$ 177$. Ideale. Teilbarkeit und Multiplikation $\ldots \ldots \ldots \ldots \ldots \ldots \ldots \ldots 116$

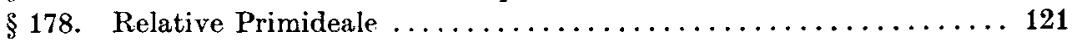

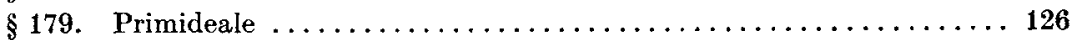

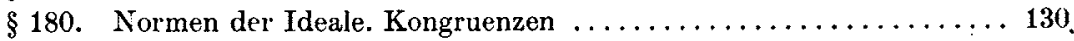

$\S 181$. Idealklassen und deren Komposition $\ldots \ldots \ldots \ldots \ldots \ldots \ldots \ldots \ldots 139$

$\$ 182$. Zerlegbare Formen und deren Komposition $\ldots \ldots \ldots \ldots \ldots \ldots \ldots 146$

$\$ 183$. Einheiten eines endlichen Körpers $\ldots \ldots \ldots \ldots \ldots \ldots \ldots \ldots \ldots 156$

$\S 184$. Anzahl der Idealklassen $\ldots \ldots \ldots \ldots \ldots \ldots \ldots \ldots \ldots \ldots \ldots \ldots \ldots 169$

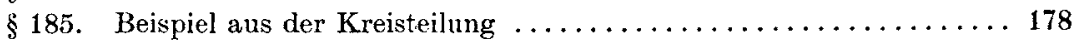

$\S 186$. Quadratische Körper .......................... 200

$\$ 187$. Moduln in quadratischen Körpern $\ldots \ldots \ldots \ldots \ldots \ldots \ldots \ldots 206$

XIVII. Über die Komposition der binären quadratischen Formen

(Supplement $X$ von Dirichlets Vorlesungen über Zahlentheorie, 2. Aufl., S. 423-462 (1871).)

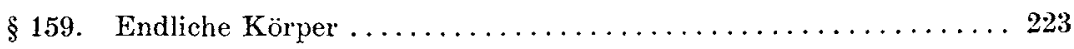

$\S 160$. Ganze algebraische Zahlen $\ldots \ldots \ldots \ldots \ldots \ldots \ldots \ldots \ldots \ldots \ldots .236$

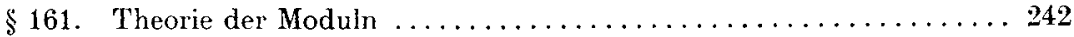

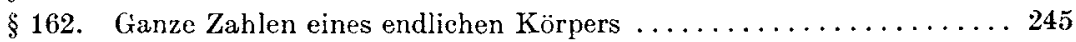

$\S 163$. Theorie der Ideale eines endlichen Körpers . . . . . . . . . 251 
XLVIII. Sur la Théorie des Nombres entiers algébriques

(Paris, Gauthier-Villars, 1877, S. 1-121. Bulletin des Sciences mathématiques et astro. nomiques, $1^{\text {Te }}$ série, t. $X I, 2^{\mathrm{e}}$ séric, t. $\left.1 ., 1876,1877.\right)$

Introduction $\ldots \ldots \ldots \ldots \ldots \ldots \ldots \ldots \ldots \ldots \ldots \ldots \ldots \ldots \ldots \ldots \ldots \ldots$

Section I. Théorémes auxiliaires de la théorie des modules $\ldots \ldots \ldots \ldots \ldots 273$

Section II. Le germe de la théorie des idéaux $\ldots \ldots \ldots \ldots \ldots \ldots \ldots \ldots \ldots$

$\$$ 5. Les nombres rationnels entiers $\ldots \ldots \ldots \ldots \ldots \ldots \ldots \ldots \ldots \ldots . \ldots 274$

$\S$ 6. Les nombres complexes entiers de Gauss ............ 276

$\$$ 7. Le domaine $\mathrm{o}$ des nombres $x+y \sqrt{-5} \ldots \ldots \ldots \ldots \ldots . \ldots 278$

$\$$ 8. Rôle du nombre 2 dans le domaine $0 \ldots \ldots \ldots \ldots \ldots \ldots$

$\S 9$. Rôle des nombres 3 et 7 dans le domaine $0 \ldots \ldots \ldots \ldots \ldots . \ldots 284$

$\$ 10$. Lois de la divisibilité dans le domaine $0 \ldots \ldots \ldots \ldots \ldots$

$\$$ 11. Idéaux dans le domaine $0 \ldots \ldots \ldots \ldots \ldots \ldots \ldots \ldots$

$\S 12$. Divisibilité et multiplication des idéaux dans le domaine 0.291

XIJX. Uber die Theorie der ganzen algebraischen Zahlen

(Supplement XI von Dirichlets Vorlesungen über Zahlentheorie, 3. Auf1., S.515-530 (1879).)

\$170. Multiplikation der Ideale $\ldots \ldots \ldots \ldots \ldots \ldots \ldots \ldots \ldots$

\$171. Relative und absolute Primideale $\ldots \ldots \ldots \ldots \ldots \ldots \ldots \ldots \ldots$

$\$$ 172. Hilfssätze . . . . . . . . . . . . . . . . . . . . . . 303

\$173. Gesetze der Teilbarkeit $\ldots \ldots \ldots \ldots \ldots \ldots \ldots \ldots \ldots \ldots$

Erläuterungen zu den vorstehenden Abhandlungen XLVI - XLIX ..... 313 